\title{
A Quantum Dot-Based Concentric FRET Configuration for the Parallel Detection of Protease Activity and Concentration
}

\author{
Miao Wu, Eleonora Petryayeva, W. Russ Algar* \\ Department of Chemistry, University of British Columbia, 2036 Main Mall, Vancouver, \\ British Columbia, V6T 1Z1, Canada. \\ *Corresponding author: algar@chem.ubc.ca
}

\begin{abstract}
Protease expression, activity, and inhibition play crucial roles in a multitude of biological processes; however, these three aspects of their function are difficult for any one bioanalytical probe to measure. To help address this challenge, we report a multifunctional concentric Förster resonance energy transfer (FRET) configuration that combines two modes of biorecognition using aptamers and peptide substrates co-assembled to a central semiconductor quantum dot (QD). The aptamer is sensitive to the concentration of protease and the peptide is sensitive to its hydrolytic activity. The role of the QD is to serve as a nanoscale scaffold and initial donor for energy transfer with both Cyanine 3 (Cy3) and Alexa Fluor 647 (A647) fluorescent dyes associated with the aptamer and peptide, respectively. Using thrombin as a model protease, we show that a ratiometric analysis of the emission from the QD, Cy3, and A647 permits discrimination between thrombin and thrombin-like activity, and distinguishes between active, reversibly inhibited, and irreversibly inhibited thrombin. Reliable quantitative results were obtained from a kinetic analysis of the changes in FRET. This concentric FRET format, which capitalizes on both the physical and optical properties of QDs, should be adaptable to other protease targets for which both peptide substrates and binding aptamers are known. It is thus expected to become valuable a tool for the real-time analysis of protease activity and regulation.
\end{abstract}




\section{Introduction}

Proteases are enzymes that hydrolyze peptide bonds and play many crucial roles in biological systems. These enzymes are highly regulated, in part through expression as zymogens, cascaded activation, and via endogenous inhibitors, so that they can function without damaging tissues or interfering with other processes. ${ }^{1}$ Approximately $80 \%$ of the digestive enzymes produced in the pancreas are proteases such as trypsin and chymotrypsin. ${ }^{2}$ Proteases including thrombin and plasmin, among several others, are also key players in hemostasis and fibrinolysis. ${ }^{3}$ Matrix metalloproteinases help regulate processes such as cell migration, differentiation, proliferation, inflammation and immunity, as well as angiogenesis, metastasis, and other processes related to cancer progression. ${ }^{4,5}$ Caspase proteases are essential to apoptosis, too little of which is associated with cancer and autoimmune disorders, and too much of which is associated with immunodeficiency and degenerative conditions such as Alzheimer's Disease. ${ }^{6}$ Many proteases are also potential drug targets; for example, secretase inhibitors have therapeutic potential in Alzheimer's disease. ${ }^{7}$ The challenge in this regard is the scope of diverse roles that continue to be uncovered for many proteases, potentially making them both drug targets and antitargets simultaneously. ${ }^{4}$ Clearly, proteases are important biological analytes, and new methods and probes for their detection and analysis are of great interest.

One of the challenges in the analysis of proteases is distinguishing between activity and concentration. The activity of enzymes is modulated by several post-translational mechanisms (e.g., inhibitors), such that their concentration is not necessarily proportional to their activity. ${ }^{8}$ Immunoassays, which are the current clinical standard, ${ }^{9}$ are generally sensitive to protease concentration and are not a direct measure of activity. Although less common than the use of antibody probes, there are at least 14 known protease-binding aptamers, ${ }^{10}$ but these assays are also sensitive to concentration rather than activity. Conversely, probes that respond to activity are not strictly sensitive to concentration. Chromogenic and fluorogenic substrates are available for many proteases, ${ }^{11}$ as are FRET-based probes utilizing peptides that are either dual-labeled with fluorescent dyes ${ }^{12}$ or singly labeled with a fluorescent dye and conjugated to an optically active nanoparticle. ${ }^{13}$ In each of these cases, there is a change in an optical signal in response to a proteolytic reaction, but not to the protease directly. A third strategy is the use of reactive probes 
that modify the active site of a protease with a tag that can be directly or indirectly labeled for quantitation. ${ }^{8}$ This approach provides a proxy measure for activity via the availability of active sites. Overall, none of the foregoing strategies measures both activity and concentration.

Here, we report a design for a fluorescent bioanalytical probe that is simultaneously sensitive to both protease activity and concentration, with the ability to resolve one from the other. The design, shown in Figure 1, takes advantage of the physical and optical properties of semiconductor quantum dots (QDs) and uses thrombin as a model protease. Poly(ethylene glycol)-coated CdSeS/ZnS QDs with peak emission at $524 \mathrm{~nm}$ (QD524) are conjugated with multiple copies of an Alexa Fluor 647 (A647) dye-labeled peptide substrate for thrombin, as well as multiple copies of a thrombin-binding aptamer partially hybridized with a Cyanine 3 (Cy3) dye-labeled oligonucleotide. The peptide is a probe for thrombin activity; the aptamer is a probe for thrombin concentration. Resolvable signals for protease activity and concentration are generated through adaptation of a concentric Förster resonance energy transfer (cFRET) configuration that we recently developed. ${ }^{14}$ The QD524 serves as a highly efficient energy donor for the $\mathrm{Cy} 3$ and a less efficient energy donor for the A647, while the Cy3 also serves as a good energy donor for the A647. ${ }^{14}$ The relative emission from the QD524, Cy3, and A647 can be calibrated to permit determination of the number of each acceptor per QD. Changes in emission are associated with proteolytic cleavage of the A647 from the QD, and with displacement of the Cy3-labeled oligonucleotide when thrombin-binding aptamer binds to thrombin, each resulting in loss of FRET pathways. These signaling mechanisms have been reported individually, ${ }^{15,16}$ but neither in combination on a common nanoparticle scaffold nor with cFRET. Whereas our previous cFRET probe was designed to detect the activity of two different proteases, this new cFRET probe is multifunctional in that it simultaneously combines two modes of biorecognition for a single protease. Active thrombin can be distinguished from inactive thrombin with reversible or irreversible inhibition of its active site, and thrombin activity can be distinguished from thrombin-like activity associated with other proteases. This strategy is therefore very promising for the bioanalysis of proteases and is uniquely facilitated by the properties of QDs. 


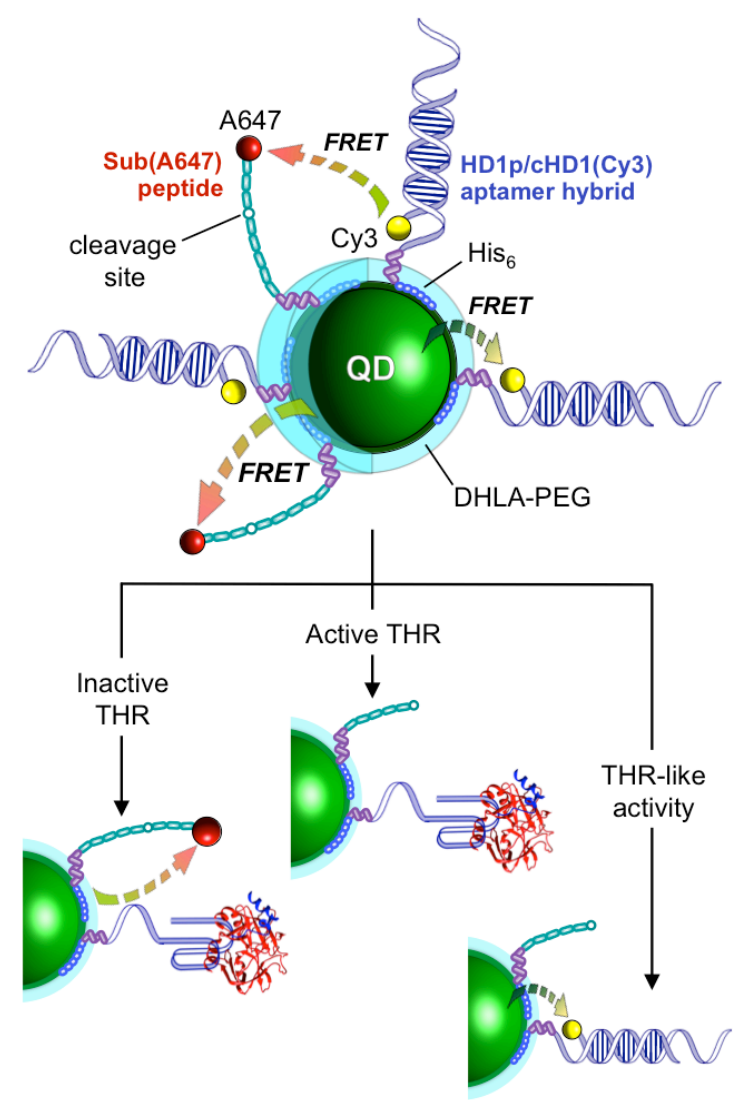

Figure 1. Schematic representation of a cFRET configuration, [HD1p/cHD1(Cy3)] ${ }_{12}$-QD524-[Sub(A647)], that can simultaneously detect thrombin (THR) activity and concentration through the use of HD1p aptamer and Sub(A647) peptide substrate probes on a common QD scaffold. (Refer to Table 1 for the aptamer and peptide sequences and abbreviations.) The configuration is designed such that active thrombin modulates all three FRET pathways, inactive thrombin (i.e., inhibited) modulates the QD-to-Cy3 and Cy3-to-A647 FRET pathways, and thrombin-like activity by other proteases modulates the QD-toA647 and Cy3-to-A647 FRET pathways.

\section{Experimental Section}

Materials. Alloyed CdSeS/ZnS QDs ( $\sim 6 \mathrm{~nm}$ diameter) with peak photoluminescence (PL) at 524 $\mathrm{nm}$ (QD524) and $624 \mathrm{~nm}$ (QD624) (Cytodiagnostics, Burlington, ON, Canada) were made water soluble by coating with poly(ethylene glycol)-appended dihydrolipoic acid (DHLA-PEG) ligands. ${ }^{17}$ Peptides (BioSynthesis Inc., Lewisville, TX, USA) were labeled with Alexa Fluor 647 $\mathrm{C}_{2}$ maleimide (Life Technologies, Carlsbad, CA, USA). ${ }^{18}$ Modified oligonucleotides were from Integrated DNA Technologies (Coralville, IA, USA) and were HPLC purified by the 
manufacturer. Peptide and oligonucleotide sequences are listed in Table 1. The thrombin-binding aptamer sequence was ligated with a polyhistidine-terminated peptide linker using disulfide exchange chemistry. ${ }^{19}$ Detailed procedures for the above reactions are available in the Supporting Information (SI). Bovine trypsin (TRP), lysozyme (LYZ), and $N_{\alpha}$-Tosyl-L-lysine chloromethyl ketone hydrochloride (TLCK) were obtained from Sigma-Aldrich (Oakville, ON, Canada). Human $\alpha$-thrombin (THR) was obtained from Haematologic Technologies (Essex Junction, VT, USA). Enzyme specifications can be found in the SI. All experiments were done in borate buffered saline (BBS; $\mathrm{pH} 8.5,50 \mathrm{mM}$ borate, $13.7 \mathrm{mM} \mathrm{NaCl}, 0.27 \mathrm{mM} \mathrm{KCl}, 3 \mathrm{mM}$ $\mathrm{MgCl}_{2}$ ).

Table 1. Peptide and oligonucleotide sequences.

\begin{tabular}{|c|c|}
\hline Peptide sequences (N- to C-terminal) $^{a}$ & Abbreviation \\
\hline Ac- $\mathrm{H}_{6} \mathrm{SP}_{6} \mathrm{GSDGNESG} L V P R \downarrow G S G C-\mathrm{A} 647$ & $\operatorname{Sub}(A 647)$ \\
\hline $\mathrm{Ac}-\mathrm{CSGP}_{5} \mathrm{GSGH}_{6}-\mathrm{Am}$ & Cys-His 6 \\
\hline Oligonucleotide sequences $^{b}$ & Abbreviation \\
\hline 5'-CCA ACA ACA GTG-3'-Cy3 & $\mathrm{cHD} 1(\mathrm{Cy} 3)$ \\
\hline $\mathrm{HSC}_{6} \mathrm{H}_{12}-5^{\prime}-\mathrm{TTC}$ ACT GT $\underline{G} G T T$ GGT GTG GTT GG-3' & HD1p \\
\hline
\end{tabular}

${ }^{a}$ The protease recognition site is indicated in italics; the hydrolysis site is indicated by the downwards arrow; Ac, N-terminal acetylation; Am, C-terminal amidation. ${ }^{b}$ The HD1 thrombin binding sequence is indicated in italics. The mismatched bases are underlined.

PL measurements. PL emission spectra and intensities were measured using an Infinite M1000 Pro plate reader (Tecan Ltd., Morrisville, NC, USA) with samples in nonbinding 96-well microtiter plate wells. The excitation wavelength was always $400 \mathrm{~nm}$, which offered negligible direct excitation of Cy3 and A647. For calibration experiments, both full PL spectra and single wavelength intensities at $524 \mathrm{~nm}, 564 \mathrm{~nm}$, and $668 \mathrm{~nm}$ were acquired (using two different measurement modes with the plate reader). For kinetic assays, PL intensities were measured at $90 \mathrm{~s}$ intervals at $524 \mathrm{~nm}, 564 \mathrm{~nm}$, and $668 \mathrm{~nm}$ for cFRET assays.

Enzyme assays. With the cFRET configuration, thrombin assays were done by tracking the A647/QD and Cy3/QD PL ratios after mixing [HD1p/cHD1(Cy3)] ${ }_{12}$-QD524-[Sub(A647)] conjugates with different concentrations of protease. To assay $n$ samples, $10 n$ pmol of 
$[\mathrm{HD} 1 \mathrm{p} / \mathrm{HD} 1 \mathrm{c}(\mathrm{Cy} 3)]_{12}-\mathrm{QD} 524-[\mathrm{Sub}(\mathrm{A} 647)]_{8}$ was prepared in $55 n \mu \mathrm{L}$ of BBS by mixing each component at the desired stoichiometry and allowing self-assembly to proceed for $4 \mathrm{~h}$. A series of dilutions of thrombin (22 nM-11.4 $\mu \mathrm{M}$, scaling by factors of two) was prepared in BBS and $50 \mu \mathrm{L}$ of each was transferred to a 96-well plate in parallel with a BBS control sample. Aliquots of $[\mathrm{HD} 1 \mathrm{p} / \mathrm{cHD} 1(\mathrm{Cy} 3)]_{12}-\mathrm{QD} 524-[\mathrm{Sub}(\mathrm{A} 647)]_{8}(50 \mu \mathrm{L})$ were added and mixed with the THR solutions. The final concentrations of THR were half of those added to the wells, and the final concentration of QDs was $91 \mathrm{nM}$. PL measurements were initiated immediately after the mixing. Assays with trypsin and lysozyme were done similarly, excepting that the final enzyme concentrations were different (1.3 nM-5.0 $\mu \mathrm{M}$ for trypsin; 0.02, 0.20,2.0 $\mu \mathrm{M}$ for lysozyme).

Inhibition assays. For these assays, probes were prepared as described above. In the case of TLCK, thrombin ( $2 \mu \mathrm{L}$ of $163 \mu \mathrm{M}$ stock solution) was first diluted with BBS (200 $\mu \mathrm{L})$ followed by the addition of TLCK in DMSO $(10 \mu \mathrm{L}, 50 \mathrm{mM})$. The mixture was let stand at room temperature for $2 \mathrm{~h}$. This procedure was repeated three times. A series of TLCK-treated thrombin solutions with concentrations between $88 \mathrm{nM}-1.4 \mu \mathrm{M}$ (scaling by a factor of two) was then prepared from the stock solution. Assays were done as described above. In the case of argatroban, a series of dilutions between 4.0-64 $\mu \mathrm{M}$ was prepared in BBS. Thrombin dilutions were also prepared in BBS at concentrations between 1.0-8.0 $\mu \mathrm{M}$. Argatroban solution $(25 \mu \mathrm{L})$ and thrombin solution $(25 \mu \mathrm{L})$ were mixed as different combinations of concentrations and added to the wells of a 96-well microtiter plate. Assays were done as described above.

Data analysis. In the cFRET configuration, PL ratios were calculated from the PL intensities measured at $524 \mathrm{~nm}, 564 \mathrm{~nm}$, and $668 \mathrm{~nm}$. At these wavelengths, the emission from the QD524, Cy3, and A647 were largely resolved, albeit that small corrections for crosstalk were required according to Eqns. 1-3, where $I_{x}$ is the intensity from emitter $x, I(\lambda)$ is the intensity measured at wavelength $\lambda$, and ${ }_{x} \sigma_{\lambda}$ is a correction factor for emission overlap, as defined in the SI (Eqns. S1S3).

$$
\begin{gathered}
I_{\mathrm{QD} 524}=I(524) \\
I_{\mathrm{Cy} 3}=I(564)-{ }_{\mathrm{QD} 524} \sigma_{564} I_{\mathrm{QD} 524} \\
I_{\mathrm{A} 647}=I(668)-{ }_{\mathrm{QD} 524} \sigma_{668} I_{\mathrm{QD}}-{ }_{\mathrm{Cy} 3} \sigma_{668} I_{\mathrm{Cy} 3}
\end{gathered}
$$

PL ratios, $\rho$, were then calculated according to Eqns. 4-5. 


$$
\begin{aligned}
\rho_{\mathrm{Cy} 3} & =I_{\mathrm{C} y 3} / I_{\mathrm{QD} 524} \\
\rho_{\mathrm{A} 647} & =I_{\mathrm{A} 647} / I_{\mathrm{QD} 524}
\end{aligned}
$$

In assays, the PL ratios were normalized to the control sample (see Eqn. S4 for details) and then converted into the number of Cy3 and A647 acceptors per QD524, $M$ and $N$ respectively, from empirical calibration functions, $\rho_{\mathrm{Cy3} / \mathrm{QD} 524}(M, N)$ and $\rho_{\mathrm{A} 647 / \mathrm{QD} 524}(M, N)$, Eqn. 6 and Eqn. 7 , derived from three-dimensional plots of $\rho_{\mathrm{Cy} 3}$ and $\rho_{\mathrm{A} 647}$ versus $(M, N)$ (see SI, Fig. S3).

$$
\begin{gathered}
\rho_{\mathrm{Cy} 3 / \mathrm{QD} 524}=(2.57)(0.032 M-0.0005)(-0.020 N+0.383) \\
\rho_{A 647 / \mathrm{DD} 524}=(4.88)(0.023 N+0.006)(0.008 M+0.111)
\end{gathered}
$$

Analytical parameters, $k$ and $\Delta$, were derived from $(M, N)$ versus time data. Aptamer progress curves, $M$ versus time, were fit with a monoexponential function, Eqn. 8 , where $A$ is an amplitude, $t$ is time, $m$ reflects the displacement kinetics, and $M_{\infty}$ is the apparent equilibrium value of $M$. When $m t$ was sufficiently small (i.e., little or no response), Eqn. 8 reduced to a linear function for data fitting (see SI for justification). Progress curve fits were reduced to a single parameter, $\Delta$, according to Eqn. 9, representing the relative change in the number of $\mathrm{cHD} 1(\mathrm{Cy} 3)$ per QD.

$$
\begin{gathered}
M(t)=A e^{-m t}+M_{\infty} \\
\Delta=A\left(A+M_{\infty}\right)^{-1}
\end{gathered}
$$

Proteolysis data, $N$ versus time, was fit with a biexponential function, Eqn. 10 , where $k_{i}$ is an empirical rate of peptide hydrolysis with amplitude $A_{i}$, and $N_{\infty}$ is the residual value of $N$. These parameters were constrained so that $N(0)$ was equal to the value calculated for the control sample. An average hydrolysis constant, $k$, was calculated using Eqn. 11.

$$
\begin{gathered}
N(t)=A_{1} e^{-k_{1} t}+A_{2} e^{-k_{2} t}+N_{\infty} \\
k=\frac{\left|-k_{1} A_{1}-k_{2} A_{2}\right|}{A_{1}+A_{2}}
\end{gathered}
$$




\section{Results}

Probe design. The cFRET probe, $[\mathrm{HD} 1 \mathrm{p} / \mathrm{cHD} 1(\mathrm{Cy} 3)]_{12}-\mathrm{QD} 524-[\mathrm{Sub}(\mathrm{A} 647)]_{8}$, incorporated several important elements into its physical design. The central QD524 donor was coated with DHLA-PEG ligands to impart robust colloidal stability. The DHLA moiety tightly binds to the $\mathrm{ZnS}$ shell of the $\mathrm{CdSeS} / \mathrm{ZnS}$ core/shell QDs and the PEG moiety resists the non-specific adsorption of many biomolecules and $\mathrm{pH}$ - or ionic strength-induced aggregation. ${ }^{17}$ Indeed, we found that even low concentrations of human $\alpha$-thrombin were able to aggregate QDs coated with DHLA or glutathione, whereas DHLA-PEG-coated QDs remained stable. The Sub(A647) peptide had an N-terminal polyhistidine peptide sequence to permit high-affinity, stoichiometric self-assembly to the ZnS shell of the QDs. ${ }^{20,21}$ The polyhistidine tag is able to penetrate the DHLA-PEG ligand coating and coordinate directly to the inorganic surface of the $\mathrm{ZnS}$ shell. ${ }^{20,21}$ Similarly, the HD1p aptamer was appended with a polyhistidine peptide (Cys-His 6 ; see Table 1) using disulfide exchange. ${ }^{19}$ Gel electrophoresis confirmed assembly of both peptide and aptamer to QDs (see Fig. S1). Sub(A647) contained the LVPRGS amino acid sequence, which is recognized and hydrolyzed by thrombin, ${ }^{22}$ resulting in changes in both QD524 $\rightarrow$ A647 and Cy3 $\rightarrow$ A647 FRET (the arrow indicates the direction of energy transfer). Thrombin has a preference for hydrolysis after arginine in the P1 position, with proline at P2 and either serine, threonine, or glycine at $\mathrm{P}^{\prime}{ }^{23}$ The HD1p aptamer was hybridized with $\mathrm{cHD} 1(\mathrm{Cy} 3)$, a partially complementary Cy3-labeled oligonucleotide, to yield changes in QD524 $\rightarrow \mathrm{Cy} 3$ and $\mathrm{Cy} 3 \rightarrow \mathrm{A} 647$ FRET upon thrombin-binding. The cHD1(Cy3) sequence was shorter than HD1p, hybridizing to only part of the thrombin-binding HD1 sequence, as reported previously. ${ }^{16}$ However, we found that a mismatch had to be incorporated to facilitate displacement of cHD1(Cy3) by thrombin. Without a mismatch, the kinetics of displacement were very slow $(>4 \mathrm{~h})$ at room temperature.

Assays with active enzymes. The cFRET probe was first tested against thrombin. Given the probe design, thrombin was expected to hydrolyze the $\operatorname{Sub}(\mathrm{A} 647)$ peptide and displace the cHD1(Cy3) oligonucleotide from the QD524 when it was bound by HD1p aptamer. Figure 2A(i) shows changes in the Cy3/QD524 and A647/QD524 PL ratios, as a function of time, when the cFRET probe was exposed to increasing amounts of thrombin $(11 \mathrm{nM}-5.6 \mu \mathrm{M})$. Decreases in these ratios reflected the loss of the QD524 $\rightarrow \mathrm{Cy} 3$ and the QD524 $\rightarrow \mathrm{A} 647$ plus $\mathrm{Cy} 3 \rightarrow \mathrm{A} 647$ 
FRET pathways, respectively. Conversion of the PL ratio data to progress curves (i.e., the approximate number of $\mathrm{Cy} 3$ and $\mathrm{A} 647$ per QD as a function time) gave the expected trends, as shown in Figure 2A(ii). This conversion was done using calibration curves that correlated PL ratios with the numbers of cHD1(Cy3) and Sub(A647) per QD (see Fig. S3). With increasing concentrations of thrombin, the rate of hydrolysis of Sub(A647) peptide increased and the equilibrium number of $\mathrm{HD} 1 \mathrm{p} / \mathrm{cHD} 1(\mathrm{Cy} 3)$ aptamer hybrids per QD decreased. Note that the interaction of thrombin with $\mathrm{HD} 1 \mathrm{p}$ is a reversible binding reaction that can reach equilibrium, whereas the hydrolysis of $\operatorname{Sub}(\mathrm{A} 647)$ by thrombin is an irreversible reaction. At higher concentrations of thrombin, the progress curves for the hydrolysis of Sub(A647) clearly converged to the same end point, consistent with expectations. The data shows that analyses can be completed within 20-60 min. 

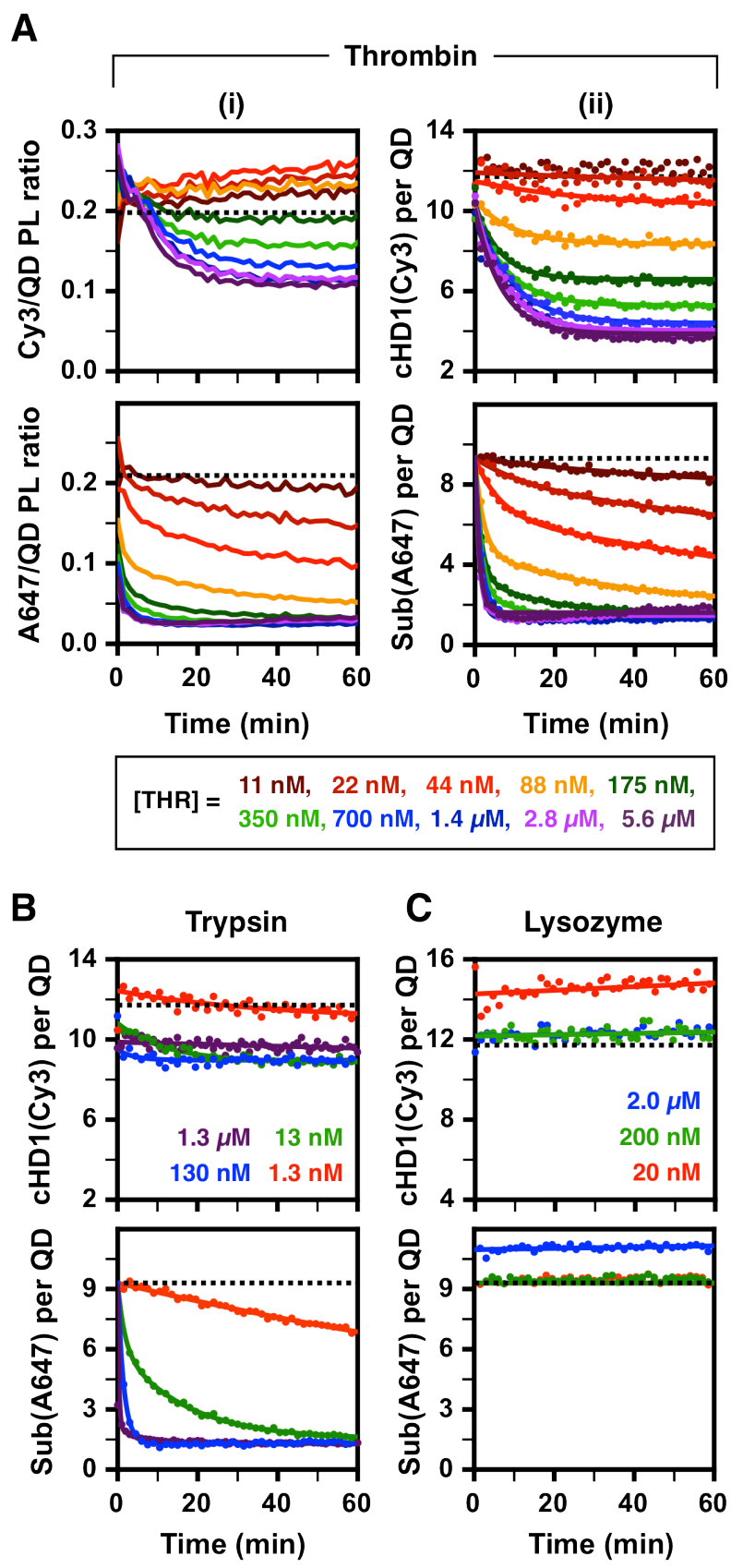

Figure 2. (A) Response of the cFRET probe to different concentrations of thrombin (THR): (i) changes in the Cy3/QD524 and A647/QD524 PL ratios as a function of time; and (ii) calculated progress curves for the binding of thrombin by HD1p aptamer with displacement of cHD1(Cy3), and hydrolysis of Sub(A647) peptide by active thrombin. (B) Progress curves showing the response of the cFRET probe to trypsin, which exhibits thrombin-like hydrolytic activity. (C) Progress curves showing the response to lysozyme, a non-proteolytic enzyme. The dashed lines are normalized control data for samples without enzyme. 
Next, the cFRET probe was tested against trypsin and lysozyme. Trypsin, like thrombin, is a serine protease that hydrolyzes peptide bonds C-terminal to arginine residues, ${ }^{24}$ and was therefore expected to exhibit thrombin-like activity toward Sub(A647) peptide but not bind with HD1p aptamer. Figure 2B shows progress curves in response to four concentrations of trypsin $(1.3 \mathrm{nM}, 13 \mathrm{nM}, 130 \mathrm{nM}, 1.3 \mu \mathrm{M}$; data for more concentrations are shown in the SI). The rate of hydrolysis of Sub(A647) peptide scaled with the concentration of trypsin, and the progress curves were clearly convergent for the highest concentrations. In contrast, the number of HD1p/cHD1(Cy3) aptamers per QD was approximately constant as a function of time, regardless of trypsin concentration, albeit with some offset (vide infra). This result demonstrated that the cFRET probe responded to thrombin-like proteolytic activity differently than it responded to thrombin, with the distinction being the minimal response from the aptamer. Finally, as a negative control, the cFRET probe was tested against lysozyme, a glycosidase that should neither hydrolyze the $\mathrm{Sub}(\mathrm{A} 647)$ peptide nor bind with the HD1p aptamer. Figure 2C shows that, consistent with expectations, the number of $\mathrm{Sub}(\mathrm{A} 647)$ and HD1p/cHD1(Cy3) were both constant over time for the three different concentrations of lysozyme (20 nM, $200 \mathrm{nM}, 2.0 \mu \mathrm{M})$.

To reduce the progress curves for hydrolysis of Sub(A647) peptide substrates and displacement of cHD1(Cy3) aptamer complements to single numerical parameters, and to help account for apparent offsets between samples, the progress curve data in Figure 2 were fit to mathematical functions (see Experimental Section, eqns. 8-11). The Sub(A647) data was reduced to an average hydrolysis constant, $k\left(\mathrm{~min}^{-1}\right)$, and the displacement of $\mathrm{cHD} 1(\mathrm{Cy} 3)$ was reduced to a relative change in the number of $\mathrm{cHD} 1(\mathrm{Cy} 3)$ per $\mathrm{QD}, \Delta$ (unitless). The progress curve data in Figure 2, summarized in terms of $k$ and $\Delta$, is shown in Figure 3. The kinetic trends observed in Figure 2 were reflected in the trends in $k$ and $\Delta$. Notably, both $k$ and $\Delta$ increased with increasing concentration of active thrombin, only $k$ increased in response to trypsin, and both $k$ and $\Delta$ were close to baseline values for lysozyme. The empirical $k$ value was found to be a more robust and more sensitive reduction of the progress curve data than calculation of initial proteolytic rates, and calculating $\Delta$ as a relative change was more robust than calculation of an absolute change. 


\section{A - Aptamer probe}

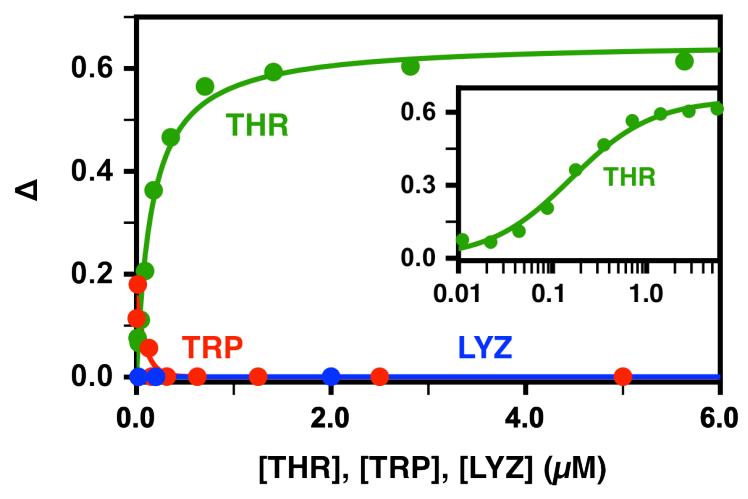

B - Peptide probe

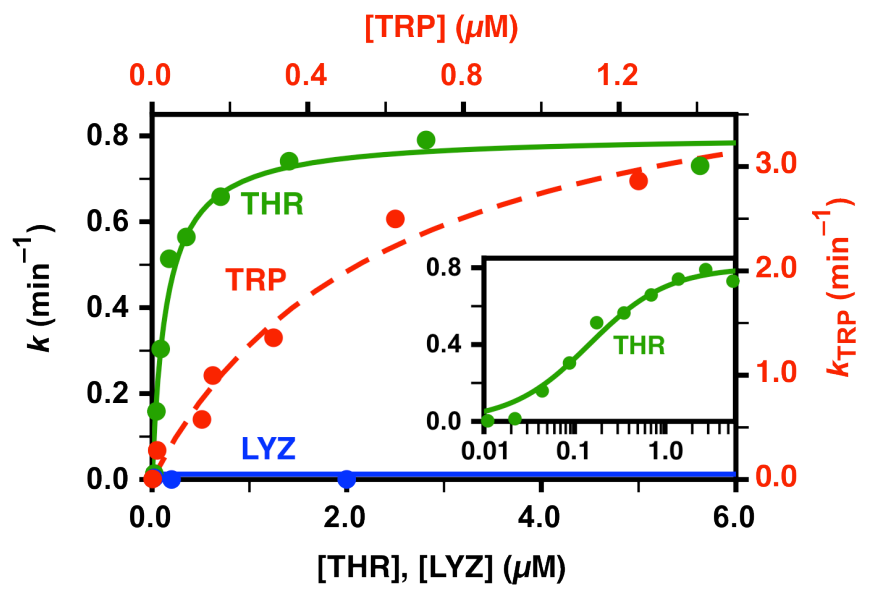

Figure 3. (A) Relative change in the number of cHD1(Cy3) per QD524, $\Delta$, and (B) the empirical hydrolysis constant, $k$, of $\mathrm{Sub}(\mathrm{A647})$ as a function of the concentration of thrombin (THR), trypsin (TRP), or lysozyme (LYZ). The insets show the responses to thrombin on a logarithmic scale. Note that the trypsin data has its own set of axes in panel B. Rates for trypsin concentrations $>1.3 \mu \mathrm{M}$ were too fast to determine.

Assays with inhibited thrombin. To further evaluate the overall selectivity of the cFRET probe, assays were done with both reversibly and irreversibly inhibited thrombin. To reversibly inhibit thrombin, argatroban was used. Argatroban is a small molecule, direct inhibitor of thrombin with clinical applications and rapid anti-thrombin action via binding with the active site of the protease; the inhibition constant is reported to be $39 \mathrm{nM} .{ }^{25}$ We tested the cFRET probe against a two-dimensional series of increasing thrombin and argatroban concentrations. An example of progress curves for this experiment are shown in Figure 4A(i) and the raw PL data can be found in the SI. Increases in the concentration of argatroban had no significant effect on the number of 
HD1p/cHD1(Cy3) aptamer hybrids per QD524 but markedly decreased the rate of hydrolysis of Sub(A647) peptide. Increases in the concentration of thrombin were able to diminish the level of inhibition from the argatroban. Figure 4A(ii) shows these trends in terms of $k$ and $\Delta$, where $k$ decreased with increasing concentrations of argatroban and increased with increasing concentrations of thrombin, and $\Delta$ was insensitive to argatroban but increased with increasing concentrations of thrombin. All of these results were consistent with expectations for reversible inhibition of thrombin at the active site.

A

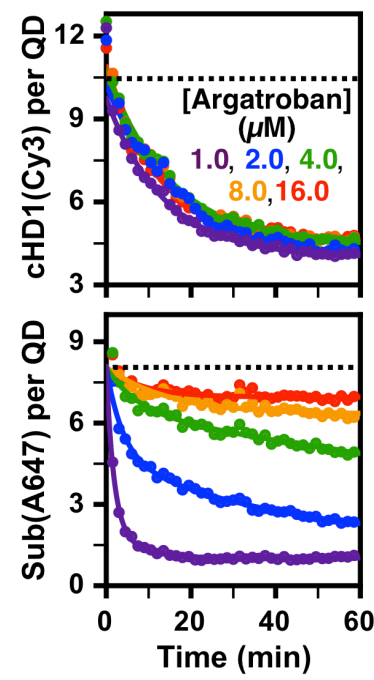

B
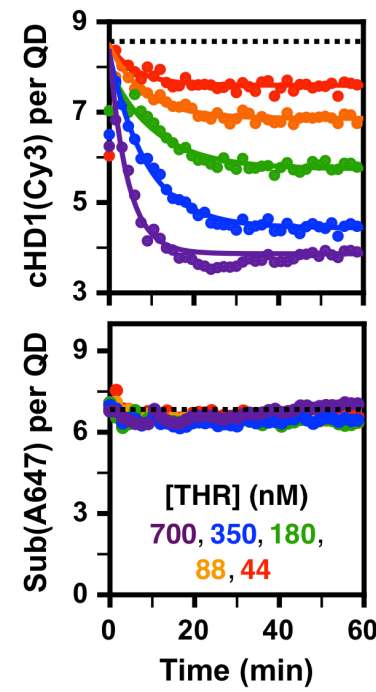

(ii)
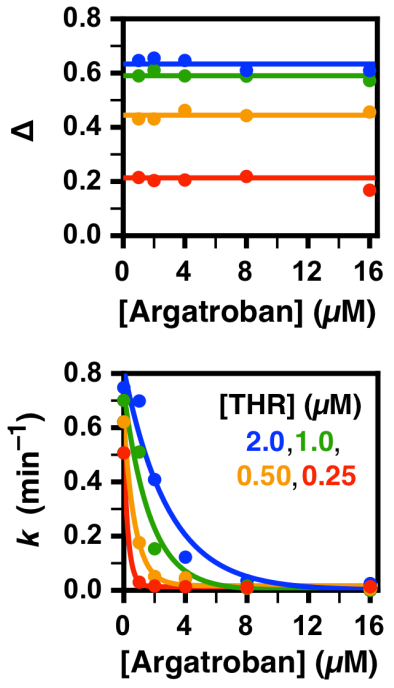

(ii)
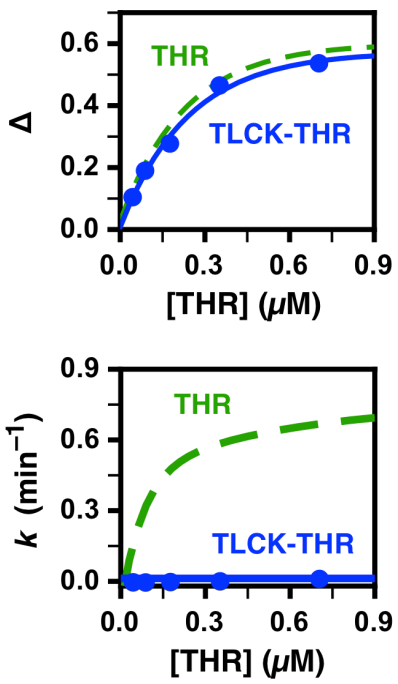

Figure 4. (A) Response of the cFRET probe to different concentrations of thrombin and argatroban, a reversible inhibitor: (i) representative progress curves for $1 \mu \mathrm{M}$ thrombin (see SI for full data set); (ii) 
summary plots showing changes in $\Delta$ and $k$ for a range of both thrombin and argatroban concentrations. The legends in (i) and (ii) apply to both plots. (B) Response of the cFRET probe to different concentrations of inactive, TLCK-treated thrombin (irreversibly inhibited): (i) progress curves; and (ii) summary plots showing changes in $\Delta$ and $k$, with comparisons to the analogous data for active thrombin. The legend in (i) applies to both plots. The dashed lines in panels A and B are normalized control data for samples without thrombin.

To irreversibly inhibit thrombin, treatment with an excess of TLCK was utilized. Unlike argatroban, which non-covalently binds to the active site of thrombin, TLCK alkylates the active site at the histidine residue in the histidine-aspartic acid-serine catalytic triad. ${ }^{26}$ Figure $4 \mathrm{~B}(\mathrm{i})$ shows progress curves for the cFRET probe in response to increasing concentrations of TLCKinhibited thrombin. In each case, no hydrolysis of Sub(A647) peptides was observed and the decrease in the number of $\mathrm{cHD} 1(\mathrm{Cy} 3)$ aptamer complements per QD524 scaled in proportion to the concentration of thrombin. This contrast was expected for complete, irreversible inhibition of thrombin at the active site. Accordingly, the value of $k$ was effectively zero for all concentrations of TLCK-thrombin whereas $\Delta$ scaled in proportion to the TLCK-thrombin concentration, as shown in Figure 4B(ii). Overall, the experiments in Figure 4 demonstrate that detection of thrombin activity and concentration is approximately independent.

Validation against a two-probe non-cFRET system. To validate the results obtained with the cFRET probe, a system with two separate QD probes was tested against a similar battery of experiments. The two probes were conventional donor-acceptors pairs, a QD524$[\mathrm{HD} 1 \mathrm{p} / \mathrm{cHD} 1(\mathrm{Cy} 3)]_{12}$ conjugate and a QD624-[Sub(A647) $]_{8}$ conjugate, which separately responded to thrombin concentration and activity, respectively, when these two discrete entities were mixed in the same sample solution. These results, which are shown in the SI, were analogous to those obtained with the cFRET probe: increasing concentrations of active thrombin decreased the number of cHD1(Cy3) per QD524 and Sub(A647) per QD624; trypsin decreased the number of Sub(A647) per QD624 and, curiously, caused a small increase in the apparent number of cHD1(Cy3) per QD; and lysozyme neither altered the number of cHD1(Cy3) per QD524 nor the number of Sub(A647) per QD624. TLCK-treated thrombin only altered the number of cHD1(Cy3) per QD524, and increasing concentrations of argatroban affected the rate of hydrolysis of Sub(A647) from QD624 but not the equilibrium number of cHD1(Cy3) per 
QD524. Cumulatively, this data confirmed that the multifunctional cFRET probe was able to perform the function of two separate QD probes.

Effect of co-assembling aptamer and peptide substrate. Given that the HD1 aptamer has been reported to be an inhibitor of thrombin activity, ${ }^{27}$ we evaluated the effect that co-localizing Sub(A647) and HD1p on a common QD scaffold had on the proteolytic activity of thrombin toward $\mathrm{Sub}(\mathrm{A} 647)$. [HD1p $]_{M}-\mathrm{QD} 524-[\mathrm{Sub}(\mathrm{A} 647)]_{8}$ conjugates were prepared with different values of $M$, and the proteolytic activity of thrombin was evaluated and compared to samples of QD524-[Sub(A647)] $]_{8}$ mixed with $M \times H D 1 p$ aptamer (without a ligated polyhistidine tag, thus avoiding assembly to the QD). As shown in Figure 5, increasing amounts of aptamer decreased proteolytic activity in both cases; however, the inhibitory effect of HD1p aptamer in bulk solution was greater than with the HD1p aptamer bound to the QD (i.e., cFRET probe). Coconjugation of the Sub(A647) peptide probe and the HD1p aptamer to a common QD scaffold appeared to partially mitigate the inhibitory effects of the latter.

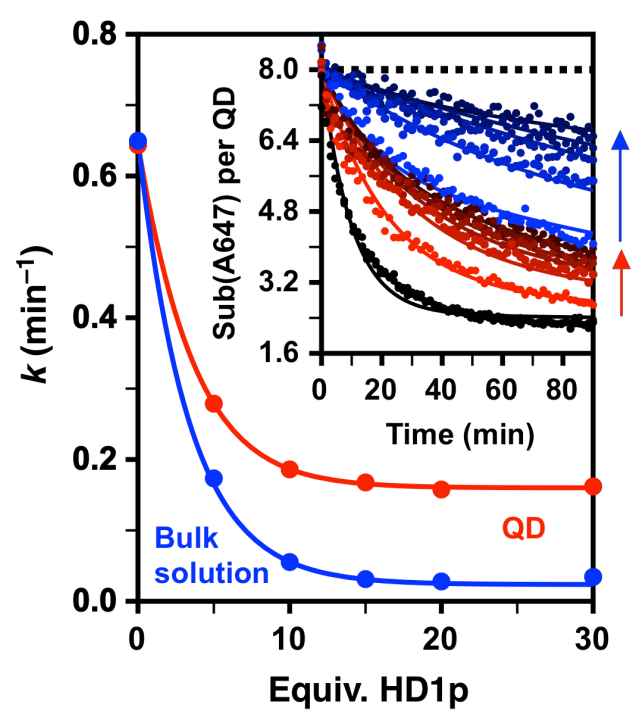

Figure 5. Change in the hydrolysis rate constant, $k$, for $[\mathrm{HD} 1 \mathrm{p}]_{M}-\mathrm{QD} 524-[\mathrm{Sub}(\mathrm{A647})]_{8}$ conjugates (QD, red points) and QD624-[Sub(A647)]8 mixed with $M \times[H D 1 p]$ aptamer (bulk solution, blue points), for $M=$ $0-30$. The inset shows the progress curves used to calculate $k$. The arrows point in the direction of increasing equivalents of HD1p aptamer per QD. The black points correspond to a control experiment without HD1p. The dashed line is the normalized control data for a sample without thrombin. 


\section{Discussion}

The cFRET probe was multifunctional, utilizing two mechanisms of biorecognition and multiple FRET pathways to signal the concentration and activity of thrombin via changes in the ratiometric PL of the QD524, Cy3, and A647. Quantitative data was obtained by pre-calibration of these PL ratios. The conversion of raw PL data to $M$ and $N$, the number of each probe per QD, was sensitive to nanomolar concentrations of thrombin and selective for concentration and activity, respectively, as designed. The PL response had some limited sensitivity toward nonspecific secondary effects as trypsin and lysozyme, in sufficient concentration, caused offsets in the progress curves. However, these offsets were static for concentrations of lysozyme and trypsin that were comparable to the concentrations of thrombin (up to $\geq 2.0 \mu \mathrm{M}$ ). Occasional batch-to-batch variation also caused the calculated initial conjugate valences to vary from the nominal $M=12$ and $N=8$ (e.g., see Fig. 4B). The effects of these variations and offsets were minimized by fitting the progress curves to obtain $\Delta$ and $k$, which were more robust analytical measurements than one-point measurements of $M$ and $N$. In terms of $\Delta$ and $k$, the response of the cFRET probe to lysozyme was negligible, and low concentrations of trypsin yielded only small values of $\Delta$, which approached zero with increasing concentration because the aforementioned offsets in PL ratio became effectively instantaneous. The reason for the small $\Delta$ response to trypsin is unclear, but trypsin also evolved a non-specific response from QD524$[\mathrm{HD} 1 \mathrm{p} / \mathrm{cHD} 1(\mathrm{Cy} 3)]_{12}$ conjugates in the two-probe non-cFRET system (see Fig. S11). Nonspecific interactions between trypsin and QDs have been previously reported to cause similar offsets in FRET ratios and calculated conjugate valences in other systems. ${ }^{28}$ The response to trypsin is thus not an artifact of the cFRET system, but rather a physical phenomenon. Given that the $\Delta$ offsets were (i) too rapid to be related to displacement of cHD1(Cy3), (ii) decreased with increases in trypsin concentration; and (iii) reached an equilibrium, all of which is inconsistent with non-specific hydrolysis, we speculate that these offsets arise from a non-specific interaction between the QDs and trypsin. In complex biological media, the high-concentration protein background is also expected to cause a static offset that does not interfere with detection via $k$ and $\Delta$. Alternatively, it should be possible to minimize or eliminate these effects, and improve precision, with further optimization of QD materials, surface chemistry, and biorecognition probes. 
The reduced inhibitory effects of HD1p aptamer on thrombin when co-conjugated to a QD scaffold with Sub(A647) substrate may be an outcome of one or more of the following factors: a dynamic binding equilibrium between the thrombin and HD1p aptamer, where thrombin dissociates and sees a high local concentration of Sub(A647) at the QD interface; reduced affinity between thrombin and HD1p aptamer when the latter is conjugated to the QD and partially hybridized with cHD1(Cy3) (our data indicates $K_{\mathrm{d}} \approx 160 \mathrm{nM}$, which is higher than the $34 \mathrm{nM}$ reported elsewhere ${ }^{29}$ ); and an enhancement of proteolytic activity when substrate is conjugated to a QD. ${ }^{30}$ The trend of a decrease in proteolytic activity with increasing numbers of HD1p aptamer per QD may be a result of greater binding between thrombin and HD1p at the expense of interactions between thrombin and $\operatorname{Sub}(\mathrm{A} 647)$, increased steric hindrance that limits the accessibility of $\mathrm{Sub}(\mathrm{A} 647)$ to thrombin, or a combination of these two factors. As the $\mathrm{Sub}(\mathrm{A647})$ peptide is much smaller than HD1p aptamer, the Sub(A647) is not expected to sterically hinder interactions between thrombin and HD1p; however, steric interactions between multiple HD1p aptamers on a common QD nanoparticle could potentially contribute to the apparent increase in the $K_{\mathrm{d}}$ value noted above. In general, the relative affinities of the aptamer for the protease, as well as the affinity of the protease for the substrate and its turnover, are likely to be important design criteria for cFRET probes of the type described here. The use of aptamers with smaller dissociation constants will likely need to be balanced by the use of peptide probes for which proteases have larger specificity constants.

The ability of the cFRET probe to discriminate between thrombin activity and concentration was predicated on the ability of the HD1p aptamer to bind inhibited thrombin. The HD1 Gquadruplex sequence binds to exosite I of thrombin, ${ }^{10}$ such that binding of argatroban and TLCK at the active site of thrombin did not affect the ability of HD1p to bind thrombin. In addition to binding at the active site, regulators of thrombin activity can also bind at exosite I or exosite II. ${ }^{31}$ Inhibitors bound at exosite I would interfere with HD1 aptamer-based detection of thrombin concentration, which may be a limitation in some experiments or provide a selectivity advantage in other experiments. Note that these considerations pertain to aptamer selection, and not the cFRET configuration itself. HD22 is another common thrombin-binding aptamer, but this sequence binds to exosite $\mathrm{II}^{10}$ and therefore would be expected to be sensitive to different 
thrombin-inhibitor complexes than HD1, providing a further consideration for the design and optimization of future cFRET probes.

The overall multifunctional probe strategy with cFRET is adaptable to other protease targets for which aptamer and substrate sequences are known by substitution of the thrombin-selective sequences. Thrombin was a good initial system for proof-of-concept because its physical and catalytic properties are well-characterized, its aptamer and substrate sequences are well-known, and it has many biological roles in addition to hemostasis (e.g., tumor biology, angiogenesis, inflammation $^{32}$ ). Ultimately, cFRET probes of this nature are expected to be highly valuable for analysis and imaging of the dynamics of many proteases in cultured cells or tissues, extracts, and other biological samples. Multifunctional sensing is achieved using a single probe entity and, in principle, retains this functionality from the ensemble down to the level of single particles, minimizing the amount of exogenous probe that must be introduced to a biological system.

\section{Conclusions}

We have demonstrated proof-of-concept for a QD-based cFRET configuration that, as a discrete probe, combines two modes of biorecognition to simultaneously and quantitatively detect both protease activity and concentration. Here, thrombin was used as a model system. An aptamer sequence was used to bind thrombin and a peptide substrate was used to measure thrombin activity. FRET signals were generated by partially hybridizing the aptamer with a Cy3-labeled oligonucleotide, and by labeling the distal terminus of the peptide with A647. A central QD524 served as a FRET donor for both of these dyes, with secondary energy transfer between Cy3 and A647. Thrombin displaced these dyes from the QD through different mechanisms, enabling the cFRET probe to distinguish thrombin activity from thrombin-like activity, and distinguish between active, partially inhibited, or completely inhibited thrombin. This format took advantage of both the physical and optical properties of QDs, and is adaptable to other protease targets for which both peptide substrates and binding aptamers are known. ${ }^{10}$ This general design for a multifunctional cFRET probe is anticipated to be a valuable tool for real-time measurements of protease activity and regulation. It provides more information than aptamer or peptide probes alone, while also obviating any need for two separate probes. The overall strategy of combining 
affinity-based and activity-based biomolecule probes on a common nanoparticle scaffold is also likely to benefit the analysis of other classes of enzyme.

\section{Acknowledgements}

The authors acknowledge financial support for this research from the Natural Sciences and Engineering Research Council of Canada (NSERC), the Canada Foundation for Innovation, and the University of British Columbia. E.P. is grateful for a postgraduate scholarship from NSERC. W.R.A. is grateful for a Canada Research Chair (Tier 2) and Michael Smith Foundation for Health Research Scholar Award.

\section{Supporting Information}

Full experimental details including DHLA-PEG ligand synthesis and application to QDs, bioconjugation and labeling reactions, additional aspects of data analysis, additional results including gel electrophoresis of the QD-bioconjugates, characterization of the FRET pairs, PL ratio data for the cFRET probe assays, and full data sets for the two-probe non-cFRET assays. This material is available free of charge via the Internet at http://pubs.acs.org.

\section{References}

(1) Twining, S. S. Crit. Rev. Biochem. Mol. Biol. 1994, 29, 315-383.

(2) Whitcomb, D. C.; Lowe, M. E. Dig. Dis. Sci. 2007, 52, 1-17.

(3) Gebbink, M. F. B. G.; Bouma, B.; Maas, C.; Bouma, B. N. FEBS Letters 2009, 583, 26912699.

(4) Dufour, A.; Overall, C. M. Trends. Pharmacol. Sci. 2013, 34, 233-242.

(5) Hadler-Olsen, E.; Winberg, J. O.; Uhlin-Hansen, L. Tumor Biol. 2013, 34, 2041-2051.

(6) Danial, N. N.; Korsmeyer, S. J. Cell 2004, 116, 205-219.

(7) De Strooper, B.; Vassar, R.; Golde, T. Nat. Rev. Neurol. 2010, 6, 99-107.

(8) Cravatt, B. F.; Wright, A. T.; Kozarich, J. W. Annu. Rev. Biochem. 2008, 77, 383-414.

(9) Wu, J.; Fu, Z.; Yan, F.; Ju, H. Trends Anal. Chem. 2007, 26, 679-688.

(10) Dupont, D. M.; Andersen, L. M.; Botkjaer, K. A.; Andreasen, P. A. Curr. Med. Chem. 2011, $18,4139-4151$.

(11) Gershkovich, A. A.; Kholodovych, V. V. J. Biochem. Biophys. Methods 1996, 33, 135-162.

(12) Lee, S.; Xie, J.; Chen, X. Curr. Top. Med. Chem. 2010, 10, 1135-1144.

(13) Kim, G. B.; Kim, Y. P. Theranostics 2012, 2, 127-138.

(14) Algar, W. R.; Ancona, M. G.; Malanoski, A. P.; Susumu, K.; Medintz, I. L. ACS Nano 2012, 6, 11044-11058.

(15) Medintz, I. L.; Clapp, A. R.; Brunel, F. M.; Tiefenbrunn, T.; Uyeda, H. T.; Chang, E. L.; Deschamps, J. R.; Dawson, P. E.; Mattoussi, H. Nat. Mater. 2006, 5, 581-589. 
(16) Levy, M.; Cater, S. F.; Ellington, A. D. Chem. Bio. Chem. 2005, 6, 2163-2166.

(17) Mei, B. C.; Susumu, K.; Medintz, I. L.; Delehanty, J. B.; Mountziaris, T. J.; Mattoussi, H. J. Mater. Chem. 2008, 18, 4949-4958.

(18) Algar, W. R.; Blanco-Canosa, J. B.; Manthe, R. L.; Susumu, K.; Stewart, M. H.; Dawson, P. E.; Medintz, I. L. Meth. Mol. Biol. 2013, 1025, 47-73.

(19) Algar, W. R.; Wegner, D.; Huston, A. L.; Blanco-Canosa, J. B.; Stewart, M. H.; Armstrong, A.; Dawson, P. E.; Hildebrandt, N.; Medintz, I. L. J. Am. Chem. Soc. 2012, 134, 1876-1891.

(20) Aldeek, F.; Safi, M.; Zhan, N. Q.; Palui, G.; Mattoussi, H. ACS Nano 2013, 7, 10197-10210.

(21) Sapsford, K. E.; Pons, T.; Medintz, I. L.; Higashiya, S.; Brunel, F. M.; Dawson, P. E.; Mattoussi, H. J. Phys. Chem. C 2007, 111, 11528-11538.

(22) Terpe, K. Appl. Microbiol. Biotechnol. 2003, 60, 523-533.

(23) Gallwitz, M.; Enoksson, M.; Thorpe, M.; Hellman, L. PLoS One 2012, 7, e31756.

(24) Vandermarliere, E.; Mueller, M.; Martens, L. Mass Spectrometry Rev. 2013, 32, 453-465.

(25) Walenga, J. M. Pathophysiol. Haemostasis Thrombosis 2004, 32(suppl 3), 9-14.

(26) Workman, E. F.; White, G. C.; Lundblad, R. L. J. Biol. Chem. 1977, 252, 7118-7123.

(27) Krauss, I. R.; Merlino, A.; Randazzo, A.; Novellino, E.; Mazzarella, L.; Sica, F. Nucleic Acids Res. 2012, 40, 8119-8128.

(28) Algar, W. R.; Malanoski, A. P.; Susumu, K.; Stewart, M. H.; Hildebrandt, N. Anal. Chem. 2012, 84, 10136-10146.

(29) Kretz, C. A.; Stafford, A. R.; Fredenbugh, J. C.; Weitz, J. I. J. Biol. Chem. 2006, 281, 37477-37485.

(30) Algar, W. R.; Malanoski, A.; Deschamps, J. R.; Blanco-Canosa, J. B.; Susumu, K.; Stewart, M. H.; Johnson, B. J.; Dawson, P. E.; Medintz, I. L. Nano Lett. 2012, 12, 3793-3802.

(31) Lane, D. A.; Philippou, H.; Huntington, J. A. Blood 2005, 106, 2605-2612.

(32) Danckwardt, S.; Hentze, M. W.; Kulozik, A. E. J. Mol. Med. 2013, 91, 1257-1271. 
Analytical Chemistry, 2014, 86, 11181-11188.

\section{TOC Graphic}

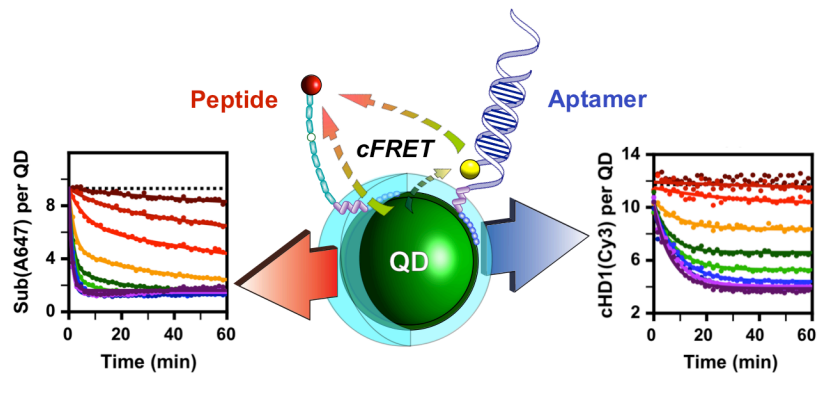

Measure Activity

Measure Concentration 